\title{
On Achromatic Probe-Forming Lenses for FIB Columns and Helium Microscopes
}

\author{
F. W. Martin
}

Nanobeam Corporation, 50 Village Ave., Dedham MA 02026

FIB columns perform poorly below $30 \mathrm{KeV}$, because chromatic aberration causes the current into a focus of constant size to decrease as the cube of the ion energy. Likewise the optimum resolution of helium microscopes [1] could be improved if chromatic aberration did not obviate use of small magnification.. Replacing the round electrostatic lens of these instruments with a doublet of achromatic quadrupoles may offer a way to overcome such limitations which is simpler than typical mid-lens correctors, as well as a way to assist in compensation of spherical aberration. Achromatic operation is well proven [2,3], but satisfactory resolution in these lenses requires compensation of second [4] and third [5] order parasitic aberrations caused by imperfect mechanical and magnetic construction [6].

An upper limit $\mathrm{P}_{4}$ to the magnitude of third-order parasitic aberration at the focal length $\mathrm{f}$ of an electric lens may be estimated by a variation of Hardy's method [6], by assuming that the electrodes of a quadrupole arrange themselves in radial displacements +-P exactly as required to produce an octopole, with the result that [7]

$$
\mathrm{P}_{4}=4 \mathrm{P} \theta^{3}
$$

where $\theta=\Delta \mathrm{R} / \mathrm{R}$ is the fraction of the lens radius $\mathrm{R}$ occupied by the ion beam.

Intentional third-order aberrations applied for compensation may be treated by expansion of the lens potential in cylindrical coordinates, and by use of the impulse approximation for the force on a particle as it travels through a lens. The deviation of a ray from its unperturbed path, caused by an octopole with the same principal plane as a single quadrupole, measured at the image distance $\mathrm{v}$ from the lenses, lies on a circle with radius

$$
\mathrm{S}_{4}=\left(2 \mathrm{~V} / \mathrm{V}_{\mathrm{A}}\right)(\mathrm{vL} / \mathrm{R}) \theta^{3}
$$

where $\mathrm{qV}_{\mathrm{A}}$ is the ion energy, $\mathrm{V}$ the octopole lens voltage, $\mathrm{v}$ the image distance, and $\mathrm{L}$ the lens length.. An important point is that the octopole field of parasites caused by random errors in pole locations may fall at any azimuth angle, and therefore compensation must have both normal and orthogonal skew (inclined at 22.5 degrees). octopoles with voltages $\mathrm{V}_{\mathrm{c}}$ and $\mathrm{V}_{\mathrm{s}}$.

The table on the next page shows computed magnitudes of compensation voltages in a single lens for three types of FIB columns. Lens dimensions are chosen to keep pole tip field and voltage below 1 tesla and 1000 volts. The uncompensated parasitic aberration of a single lens is calculated by Eq.(1) with $\mathrm{P}=2 \mu \mathrm{m}$. The compensating voltage to eliminate this parasitic aberration at the focal length of the lens is calculated by setting Eqs. $(1,2)$ equal. For helium the DC voltage of 0.9 volts cancels an uncompensated aberration of $2.3 \mathrm{~nm}$.

The doublet magnifies aberrations of its upstream lens. When normal and skew octopoles are combined with a doublet of achromatic quadrupoles, third order aberrations have the form [7] 


$$
\begin{aligned}
& x=A a^{3}+3 B a^{2} b+3 C a b^{2}+D b^{3} \\
& y=B a^{3}+3 C a^{2} b+3 D a b^{2}+E b^{3}
\end{aligned}
$$

There are only 5 coefficients to be adjusted, a considerable simplification relative to many mid-column correctors. E is by far the largest coefficient, typically as large as 100 times $\mathrm{U}_{\text {oc }}$, the strength of the normal octopole in the upstream lens. Because E is so magnified, Table 1 shows that uncompensated operation with $\mathrm{P}_{4}=2.3 \mathrm{~nm}$ cannot improve the present optimum resolution of $0.3 \mathrm{~nm}$ [1] in helium microscopy. However stabilization to 28 microvolts in the DC compensation voltage of about 1 volt produces a component of aberration (due to parasites only) of $0.01 \mathrm{~nm}$.

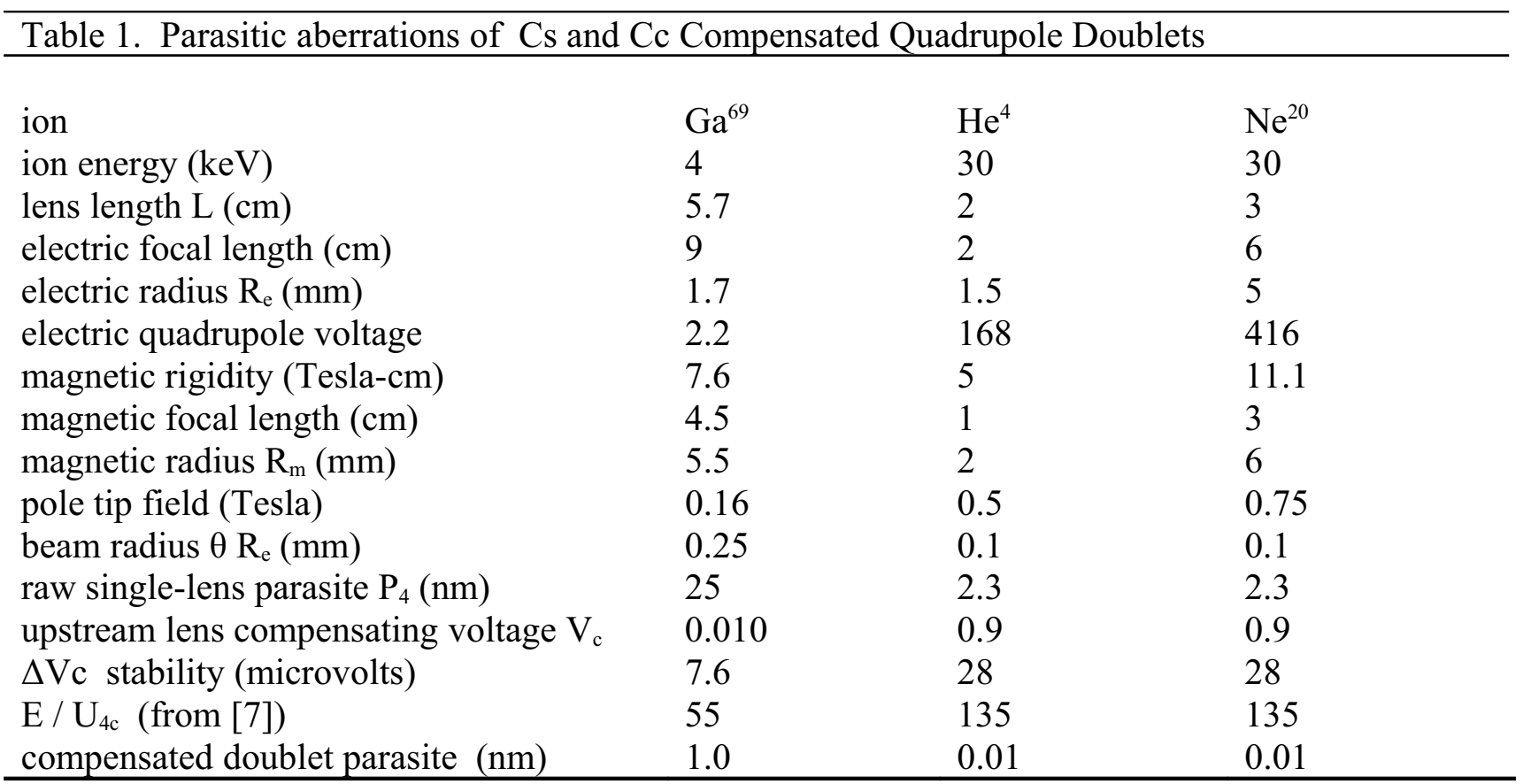

In actuality the inherent aberrations of the condenser and perfectly symmetric doublet cannot be neglected, but elimination of the parasites should allow them to be exactly compensated as described elsewhere, if a third normal mid-lens octopole is added [7]. Slow experiments show that suitable methods exist to adjust each of the 5 coefficients to zero [8]. Thus the fast measurement methods used to overcome construction tolerances for mid-column correctors - i.e. rapid image processing and accurate, stable voltage supplies -- may allow use of achromatic quadrupoles as probe-forming lenses, simpler to align and less costly than a mid-column corrector system.

[1] R. Hill, J. A. Notte, L. Scipioni, Adv. Imaging \& Elect. Phys. 170 (2012), p. 77

[2] F. W. Martin and R. Goloskie, Nucl. Instr. and Meth. in Phys. Res B54 (1991), p. 64

[3] F. W. Martin, EIPBN (2012), abstract PO7-08

[4] F. W. Martin and R. Goloskie, Nucl. Instr. And Meth in Phys. Res.B30 (1998), p 242

[5] F. W. Martin and R. Goloskie, Nucl. Instr. And Meth in Phys. Res.B104 (1995), p. 59.

[6] P. W. Hawkes, Quadrupoles in Electron Lens Design (Academic, NY) p. 65, 60

[7] F. W. Martin, submitted to Microscopy and Microanalysis; see also this conference

[8] F. W. Martin, submitted to Optik 